\title{
King's Framework and Theory in Japan, Sweden, and the United States
}

\author{
Maureen A. Frey, Liselotte Rooke, Christina Sieloff, \\ Patricia R. Messmer, Tomomi Kameoka
}

In this article, we present our work in extending and testing Imogene King's conceptual framework and theory in Japan, Sweden, and the United States. Comparing and contrasting cultural relevance, methodology, and issues of validity provide examples of international scholarship and knowledge development that result from networking and collaboration.

I nternational scholarship has been identified as an important strategy in developing and disseminating nursing knowledge (Stevenson, 1988). In addition to advancing an international research agenda, there has been exciting work done in the area of theory development. The recent International Nursing Research Congress, sponsored by Sigma Theta Tau International in Madrid, Spain, offered a group of nurse educators, researchers, and theorists an opportunity to present their work extending and testing Imogene King's Systems Framework for Nursing and Theory of Goal Attainment in Japan, Sweden, and the United States. In this article we address several important areas in knowledge development. First is the relevance, appropriateness, and utility of King's perspective for diverse cultures. The question of transcultural use has been raised in relation to King's framework specifically, and nursing theory generally. Second, is to compare and contrast the methodologies used. Third, is to evaluate the contribution to advancement of the framework and theory. These latter two areas include examples of concept development and practice applications, as well as empirical research. We conclude with recommendations for future collaboration. Our aim is to demonstrate the contribution of international scholarship and networking to nursing science.

\section{King's Conceptual Framework and Theory of Goal Attainment}

King has presented and refined her theoretical perspective for nursing in books, articles, and professional presentations over the past 25 years. Beginning with the intent of developing a framework for nursing curriculum, King asked and answered a series of critical questions: What are nursing acts, processes, and goals? Who are nurses and how are they educated for practice?
How and where is nursing practiced? Who needs nursing in this society? Based on extensive literature review, critical thinking skills, and inductive and deductive processes, the result was a conceptual framework for nursing (King, 1971).

King's $(1971,1981)$ conceptual framework, which she refers to as the Systems Framework for Nursing, consists of three open interacting systems: individuals as personal systems, small groups of individuals forming interpersonal systems, and large groups with common goals as social systems. Many concepts are identified by King that provide the basis for understanding each system and the many interactions among them.

According to King (1988), concepts describe structure and provide building blocks for theories. Theories are tested by means of research and the knowledge is used in practice. The Theory of Goal Attainment was derived by King $(1981,1991)$ from personal and interpersonal systems. Concepts in the theory are perception, growth and development, interaction, communication, transaction, role, self, coping, stress/stressors, time, and personal

Maureen A. Frey, RN, PhD, Lambda and Rho, is Assistant Professor and Assistant Research Scientist, University of Michigan School of Nursing, Ann Arbor, Michigan. Liselotte Rooke, RN, RNT, PhDr, Nursing Director, Lund University, Care Research Unit, Lund, Sweden. Christina Sieloff, RN, MSN, CNA, is Visiting Instructor, Oakland University, Rochester, Michigan. Patricia R. Messmer, RN, C, PhD, is Director of Nursing Research, Mount Sinai Medical Center, Miami Beach, Florida. Tomomi Kameoka, MN, RN, Shiga Medical University Hospital, Shiga-Ken, Japan. A symposium by this title was presented at Sigma Theta Tau International's Sixth International Nursing Research Congress in Madrid, Spain, 1993. We gratefully acknowledge Dr. Imogene King's participation in the symposium and her support of our work. Correspondence to Dr. Frey at University of Michigan School of Nursing, 400 North Ingalls, Ann Arbor, MI 48109-0482.

Accepted for publication November 10, 1993.

Image: Journal of Nursing Scholarship, 1995; 27(2), 127-130. (01995, Sigma Theta Tau International. 
space (Frey, in press). King also specified a model of transactions as the major process component of the theory. The model of transactions includes the following classification of observable behaviors: action, reaction, disturbance, mutual goal setting, exploration of means to achieve goals, and transactions (King, 1991).

King's conceptual framework was presented in the literature in the mid 1960s and early 1970s (e.g., King, 1964, 1968, 1971). The Theory of Goal Attainment was published in 1981. Although clarification, refinement, and minor changes in concepts have been made, the essential structure has been stable over time (King, 1991, 1994). King's conceptual framework and theory have been included in many texts that address nursing theory and she has been recognized as a major nurse theorist throughout the world.

Dissemination of King's ideas have at least a 20-year history in Japan. Her 1968 article, "A Conceptual Frame of Reference for Nursing," was translated and published in the Japanese Journal of Nursing Research in 1970 (Kobayashi, 1970). Her theory books (King, 1971, 1981) were transiated into Japanese and published in 1976 and 1985. Nursing theory is integrated into academic programs in Japan. Students, therefore, have the opportunity to study King's conceptual framework and theory.

The history of theory in nursing in Sweden is shorter. It was stimulated by the mandate, in 1982 , to integrate nursing conceptual frameworks into nursing education. Unlike the situation in the United States, these mandates were at the national level and directly in response to educational and healthcare reforms (Rooke, 1993). According to Rooke, it was natural to look beyond Sweden's national boundaries to the United States for leadership in nursing theory development.

\section{Cultural Considerations}

One of the interesting debates about conceptual and theoretical structures is relevance across cultures. Primary issues are cultural sensitivity and applicability. King $(1993,1994)$ states that her conceptual framework and theory are not culture bound. This is clearly supported by the value placed on an individual's subjective meaning, especially in relation to perception and health, two of her major concepts. At the same time, King does not suggest that use should be universal. Rather, use and interpretation would vary based on cultural considerations of clients and nurses. In view of this, King's conceptual framework and theory have potential for application across national and international boundaries. Others, however, (Draper, 1993; Mandelbaum, 1991) disagree with using nursing theory across cultures, challenging the assumption that a conceptual model can be applied to a practice setting other than the one for which it was developed.

Our colleagues from Japan and Sweden cited cultural relevance of King's framework and theory for nursing in their countries as major reasons for its selection. In Japan, this was primarily related to goal setting (Matsuki, 1986). In Sweden, the cultural connection was in the philosophical orientation toward person and person-environment interaction. Health care in Sweden is based on belief in the individual's integrity and autonomy. Further, guidelines for nursing practice (The National Board of Health and Welfare, 1990) identify the main task for nurses is setting goals with patients. Kameoka and Sugimori (1993) and Rooke (1993) consider cultural implications when using, interpreting, and modifying King's theoretical structures in their countries.

\section{Methodologies}

If conceptual frameworks are to provide broad structures for knowledge development, a range of methodologies and practice applications should be possible as long as approaches are consistent with the philosophic underpinnings of the frameworks. Although several sets of deductive guidelines for testing nursing frameworks have been reported (Action, Irvin, \& Hopkins, 1991; Silva, 1986), many nurses are advocates of other inductive and qualitative approaches (e.g., Benner, 1984; Carper, 1978; Fawcett, 1989, 1992; Fitzpatrick \& Whall, 1989; Silva \& Sorrell, 1992). Extending, examining, and validating conceptual frameworks will become more fruitful for the discipline with increased acceptance of the value and worth of multiple methods.

The authors' advancement of King's framework and theory can be classified according to several of Whall's (1989) categories of empirical examination of conceptual frameworks. These categories include research about concepts within the conceptual framework, examining statements derived from conceptual frameworks in research, and examining conceptual frameworks in practice. According to Whall, all these approaches, over time and taken together, will contribute to nursing knowledge.

Rooke (1993), Sieloff (1993), and Kameoka and Sugimori (1993) are working with various concepts in King's framework. Rooke has studied the concept of space using the critical incident method described by Benner (1984) and based on earlier work by Flanagan $(1949,1954)$. Nurses were asked to write narratives from their daily practice related to space. The narratives were then classified according to Hall's (1966) categories of intimate, personal, and social space. Analysis of the narratives showed that both protection and violation of space influenced patient behaviors as well as the effectiveness of nursing care. Based on the findings, Rooke proposes specific strategies for managing and controling space in nursing practice.

Sieloff (1993) is studying power and has proposed a theory of departmental power for nursing developed by reformulation and synthesis strategies. The strategic contingencies' theory of power by Hickson, Hinings, Lee, Schneck, and Pennings (1971) was reconceptualized within King's framework. Synthesis, based on the literature review, empirical observations, and components of King's perspective of power and systems relationships, were used to further specify the theory for nursing. Sieloff has formulated hypotheses designed to validate the theory.

Kameoka (Kusaka, 1991; Kameoka \& Sugimori, 1992) has conducted several studies on the process of interaction by replicating one of Dr. King's original studies. Nonparticipant observation techniques were used to examine the transaction process, which includes action, reaction, disturbance, mutual goal 
setting, exploration of means to achieve a goal, agreement on means to achieve a goal, transaction, and attainment of a goal. Kameoka and Sugimori (1993) reported on a study in which 19 cases were reviewed to determine if factors that interfered with goal attainment could be identified. Analysis resulted in several themes that described situations where goal attainment did not occur: (a) differences of perception and inadequate communication between nurse and patient, (b) one-sided (nurse directed) nurse-patient relationships, (c) lack of concern for patients on the part of nurses, and (d) nurses' lack of specific knowledge for practice.

Frey used King's conceptual framework to derive a theory of families, children, and chronic illness. Drawing from King's underlying assumption that the focus of nursing is human interaction with the environment which influences health, Frey began by examining parent and child social support (an interaction) and several child health indicators specific to families and children with insulin-dependent diabetes mellitus (IDDM). The findings of the initial study (1989) were used to expand the concepts in the theory, clarify the relationship between illness and health, and examine the use of multiple indicators of concepts. A second study, again with children with IDDM, identified an important link between health behavior and illness management behaviors (self-care). A revised theory was specified and tested with children with IDDM and a comparison sample of children with asthma. These findings showed an interesting and varied pattern of family characteristics, social support, and behavioral variables that predicted $15 \%$ to $49 \%$ of the variance in health for youths with IDDM and $16 \%$ to $72 \%$ of the variance of health for children with asthma (Frey, 1993). Theory revision and testing to include more concepts related to understanding the personal systems of children is underway.

In 1989, Messmer (1993) became involved in implementing theory based practice utilizing King's Theory of Goal Attainment at a large university-affiliate-d hospital. Economic and regulatory demands to document quality nursing care and improved patient outcomes were the impetus for the project. Planning and implementation were done with a team approach that included nursing administrators, nurse educators, clinical specialists, staff nurses, and a nurse analyst. All aspects of the nursing process were revised based on King's (1981) framework and theory. Anecdotal data from nurses indicated that theory-based practice resulted in improved care. Additionally, quantitative data demonstrated a significant increase in level of patient satisfaction. Systematic evaluation continues.

\section{Contributions to Theory Development}

Several nurse scholars (Whall, 1989; Fawcett, 1989, 1992; Silva \& Sorrell, 1992) have offered guidelines for evaluating contributions made by nursing frameworks through extension, testing, and application in practice. These guidelines balance congruency with the original structure, openness to interpretation, and freedom for development in new and creative ways (Whall, 1989). Each of the authors incorporate a variety of considerations in evaluating their work. Primary considerations are those of validity of the conceptual framework and theory followed by implications for practice, education, and research.

Kameoka and Sugimori (1993) have contributed to the validity of King's model of transactions that lead to goal attainment by examining specific ways interactions fail when transactions do not occur. The findings provide direction for teaching the process of nursing. Replication of Kameoka and Sugimori's study in the United States or in other countries would be a major contribution to understanding interaction and identification of cultural similarities and differences. One of the major contributions made by Kameoka and Sugimori is their affirmation of the cultural relevance of King's theory in Japan. It is important to hear this from our international colleagues who actively work with and study the framework and theory.

One of the advantages of using a conceptual framework is that it provides a focus and perspective consistent with the professional and social goals of the discipline. Sieloff (1993) noted that nursing uses perspectives of power from other disciplines, many of which should not be consolidated because of different philosophical bases. King's (1981) framework was selected because it can be used with a system, such as a nursing department, within the broader context of the health care system, as the study focus. Quantitative hypotheses to test the theory were presented by Sieloff. However, other qualitative methodologies could also be used to explore perceptions, understanding, and use of power by nurses. Sieloff's work is a model for reformulation of theories from other disciplines that are used by nurses.

Frey (1993) used King's (1981) conceptual framework to develop and test a middle-range theory. Knowledge derived from research has been used to modify and expand the theory. In addition, knowledge of factors that influence child health outcomes can be used to develop and test interventions. Empirical data about the effectiveness of nursing care based on King's framework would be a major contribution to the validity of the framework.

Rooke (1993) uses several approaches to evaluation, all of which are consistent with her qualitative methodology. Rooke notes that use of personal experience, as a way of scientific knowing, has been under used in nursing because it is not objective. Validity based on narrative statements is subject oriented (Sandelowski, 1986, 1991). It is the informants' experiences of the phenomena that are important. Validity was demonstrated by nurses spontaneously recognizing examples of the same concept in each other's narratives. Silva \& Sorrell (1992) note that the criteria of correspondence is the method most often used by nurses for testing and evaluating theories. Rooke demonstrates the use of criteria of coherence and pragmatism in validating truth.

Although there has always been value attached to theory-based practice, application of nursing theories and conceptual frameworks to practice have not always been recognized as useful in terms of knowledge development (Whall, 1989). Recently, Fawcett (1992) described the reciprocal relationship between conceptual models and practice. That is, models shape practice in specific ways, and practice provides data for determining the credibility of conceptual structures. Many of 
Fawcett's (1992) ideas are reflected in our work, but are especially evident in Messmer's (1993) work with the implementation of theory-based practice in a hospital setting. Messmer demonstrates how a conceptual framework can provide structure and content for patient care and proposes many points to consider in implementing that care. Plans to formally evaluate outcomes will contribute to validity of the theory.

\section{Future Directions}

New and exciting means to develop the scientific basis of nursing are likely as we move into the twenty-first century. Our work spans three countries and is directed toward extending and advancing Imogene King's conceptual framework and Theory of Goal Attainment through development of theories from the framework and descriptive efforts to better understand and use knowledge of concepts in research and practice. Discussion and debate with and by international colleagues is extremely important in shaping knowledge development. Next steps include cross-cultural research, engaging more people in theory development, extending our international network focused on King's framework and theory, and forming groups interested in developing new frameworks and theories from truly international perspectives. $\Sigma \Theta T$

\section{References}

Action, G.J., Irvin, B.L., \& Hopkins, B.A. (1991). Theory-testing research: Building the science. Advances in Nursing Science, 14(1), 52-61.

Benner, P. (1984). From novice to expert: Excellence and power in clinical nursing practice. Menlo Park, CA: Addison-Wesley.

Carper, B.A. (1978). Fundamental patterns of knowing in nursing. Advances in Nursing Science, 1(1),

13-23.

Draper, P. (1993). A critique of Fawcett's conceptual models and nursing practice: The reciprocal relationship. Journal of Advanced Nursing, 18, 558564.

Fawcett, J. (1989). Analysis and evaluation of conceptual models of nursing (2nd ed.). Philadelphia: F.A. Davis.

Fawcett, J. (1992). Conceptual models and nursing practice: The reciprocal relationship. Journal of Advanced Nursing, 17, 224-228.

Fitzpatrick, J.J., \& Whall, A.L. (Eds.). (1989). Conceptual models of nursing: Analysis and application ( 2 nd ed.). Norwalk, CT: Appleton \& Lange.

Fitzpatrick, J.J., \& Whall, A.L. (1989). Guidelines for analysis of nursing's conceptual models. In J. Fitzpatrick \& A. Whall (Eds.), Conceptual models of nursing: Analysis and application (2nd ed.) (23-31). Norwalk, CT: Appleton \& Lange.

Flanagan, J. (1949). Critical requirements: A new approach to employee evaluation. Personnel Psych, 2, 419-425.

Flanagan, J. (1954). The critical incident technique. Psychological Bulletin, 51(4), 327-358.

Frey, M.A. (1989). Social support and health: A theoretical formulation derived from King's conceptual framework. Nursing Science Quarterly, 2, 138-148.

Frey, M.A. (1993). A theoretical perspective of family and child health derived from King's conceptual framework for nursing: A deductive approach to theory building. In S.L. Feetham, S.B. Meister, J.M. Bell, \& C.L. Gilliss (Eds.), The nursing of families (30-37). Newbury Park, CA: Sage Publications, Inc.

Frey, M.A. (1993, June). Advancing King's conceptual framework for nursing and theory of goal attainment in Sweden, Japan, and the United States. Paper presented at the meeting of Sigma Theta Tau International Sixth International Nursing Research Congress, Madrid, Spain.
Frey, M.A. (in press). King's systems framework for nursing. In J.J. Fitzpatrick \& A.L. Whall (Eds), Conceptual models of nursing: Analysis and application ( $3 \mathrm{rd} \mathrm{ed}$.).

Hall, E.T. (1966). The hidden dimension. Garden City, NY: Doubleday \& Co.

Hickson, D.J., Hinings, C.R., Lee, C.A., Schneck, R.E., \& Pennings, J.M. (1971). A strategic contingencies' theory of intraorganizational power. Administrative Science Quarterly, 16, 216-229.

Kameoka, T., \& Sugimori, M. (1992). Application to the King's goal attainment theory in Japanese clinical setting. Part 2. First International Nursing Research Conference, Tokyo, 1992, proceedings, 13-14.

Kameoka, T., \& Sugimori, M. (1993, June). Application of King's goal attainment theory in Japanese clinical setting. Paper presented at the meeting of Sigma Theta Tau International Sixth International Nursing Research Congress, Madrid, Spain.

King, I.M. (1964). Nursing theory-problems and prospect. Nursing Science Quarterly, 2, 394-403.

King, I.M. (1968). A conceptual frame of reference for nursing. Nursing Research, 17, 27-31.

King, I.M. (1971). Toward a theory for nursing. New York: John Wiley \& Sons.

King, 1.M. (1981). A theory for nursing: Systems, concepts, process. New York: John Wiley \& Sons.

King, I.M. (1988). Concepts: Essential elements of theories. Nursing Science Quarterly, 1(1), 22-25.

King, I.M. (1991). King's theory of goal attainment. Nursing Science Quarterly. 5(1), 19-26.

King, I.M. (1993, June). King's conceptual system and theory of goal attainment. Paper presented at the meeting of Sigma Theta Tau International Sixth International Nursing Research Congress, Madrid, Spain.

King, I.M. (1994). Quality of life and goal attainment. Nursing Science Quarterly, 7(1), 29-32.

Kobayashi, F. (1970). A conceptual frame of reference for nursing [Translated from King, I. (1968). A conceptual frame of reference. Nursing Research, 17, 27-31]. Japanese Journal of Nursing Research, 3(3), 199-204.

Kusaka, T. (1991). Application to the King's goal attainment theory in Japanese clinical setting. Journal of Japan Academy of Nursing Education, 1(1), 30 31.

Mandelbaum, J. (1991). Why there cannot be an international theory of nursing. International Nursing Review, 38(2), 53-55, 48.

Matsuki, M. (1986). A book review: King's nursing theory. The Japanese Journal of Nursing Education, 27(3), 204.

Messmer, P. (1993, June). Implementation of theory-based nursing practice in a large teaching hospital. Paper presented at the meeting of Sigma Theta Tau International Sixth International Nursing Research Congress, Madrid, Spain.

The National Board of Health. (1990). General guidelines for nursing care in health services (no. 15). Stockholm: Liber.

Rooke, L. (1993, June). Implementing of theory-based nursing practice at a hospital for geriatric care. Paper presented at the meeting of Sigma Theta Tau International Sixth International Nursing Research Congress, Madrid, Spain.

Rooke, L. (1995). Focusing on King's theory and systems framework in education by using an experiential learning model: A challenge to improve the quality of nursing care. In M.A. Frey \& C. Sieloff (Eds.), Advancing King's framework and theory for nursing (278-293). Newbury Park, CA: Sage Publications, Inc.

Sandelowski, J. (1986). The problem of rigor in qualitative research. Advances in Nursing Science, 8(3), 27-37.

Sandelowski, J. (1991). Telling stories: Narrative approaches in qualitative research. Image: Journal of Nursing Scholarship, 23, 161-166.

Sieloff, C. (1993, June). Development of a theory of departmental power within Imogene King's framework. Paper presented at the meeting of Sigma Theta Tau International Sixth International Nursing Research Congress, Madrid, Spain.

Silva, M.C. (1986). Research testing nursing theory. Advances in Nursing Science, $9(1), 1-11$.

Silva, M.C., \& Sorrell, J.M. (1992). Testing of nursing theory: Critique and philosophical expansion. Advances in Nursing Science, 14(4), 12-23.

Stevenson, J.S. (1988). Nursing knowledge development: Into era II. Journal of Professional Nursing, 4, 152-162.

Whall, A.L. (1989). Nursing science: The process and products. In J. Fitzpatrick \& A. Whall (Eds.), Conceptual models of nursing: Analysis and application (2nd ed.) (1-14). Norwalk, CT: Appleton \& Lange. 